\title{
Membrane PD-L1 expression and soluble PD-L1 plasma levels in idiopathic pulmonary fibrosis - a pilot study
}

\author{
Dragana Jovanovic ${ }^{1,2}$, Marina Roksandic Milenkovic ${ }^{1,2}$, Jelena Kotur Stevuljevic ${ }^{3}$, Jelena Markovic ${ }^{1}$, \\ Vesna Ceriman $^{1}$, Milica Kontic ${ }^{1,2}$, Vesna Skodric Trifunovic ${ }^{1,2}$ \\ ${ }^{1}$ University Hospital of Pulmonology, Clinical Center of Serbia, Belgrade, Serbia; ${ }^{2}$ School of Medicine, University of Belgrade, Belgrade, Serbia; \\ ${ }^{3}$ Department for Medical Biochemistry, Faculty of Pharmacy, University of Belgrade, Belgrade, Serbia \\ Contributions: (I) Conception and design: D Jovanovic; (II) Administrative support: J Kotur Stevuljevic, M Roksandic Milenkovic, M Kontic, V \\ Ceriman; (III) Provision of study materials or patients: D Jovanovic, J Markovic, J Kotur Stevuljevic, M Roksandic Milenkovic; (IV) Collection and \\ assembly of data: D Jovanovic, J Markovic, J Kotur Stevuljevic, V Skodric Trifunovic, M Roksandic Milenkovic; (V) Data analysis and interpretation: \\ J Kotur Stevuljevic, D Jovanovic; (VI) Manuscript writing: All authors; (VII) Final approval of manuscript: All authors. \\ Correspondence to: Prof. dr Dragana Jovanovic. University Hospital of Pulmonology, Clinical Center of Serbia, Koste Todorovića 26, 11000 Belgrade, \\ Serbia. Email: draganajv@yahoo.com.
}

\begin{abstract}
Background: Idiopathic pulmonary fibrosis (IPF) has common risk factors with cancer and significant similarities in the pathobiology process, both diseases having poor outcomes. Immune checkpoint PD-L1 has become the target of checkpoint inhibitory therapy that unleashes antitumor $\mathrm{T}$ cells and has revolutionized cancer treatment. This is a pilot study exploring membrane immune checkpoint PD-L1 expression in human IPF lung tissue samples and its soluble form, soluble PD-L1 (sPD-L1) plasma concentrations in IPF patients, in order to investigate potential role of PD-L1 as an IPF biomarker.

Methods: Twelve human IPF lung tissue samples (formalin-fixed, paraffin-embedded) obtained by surgical biopsy, have been tested for PD-L1 expression by PD-L1 IHC 22C3 pharmDx assay, while plasma samples for examination of sPD-L1 forms, PD-L1 (B7-H1/CD274) blood concentration, originated from 23 patients with IPF who did not undergo surgical biopsy.

Results: Membrane PD-L1 expression in IPF lung tissue samples was positive to overexpression of PD-L1 in 9 samples out of 12 . Only very few cells in the interstitium have shown a discrete PD-L1 expression, but not of a membrane type. As for sPD-L1 forms, we have found elevated concentrations of sPD-L1 in the serum of IPF patients $314.3 \mathrm{ng} / \mathrm{L}(117.7-483.1 \mathrm{ng} / \mathrm{L})$, significantly higher compared with healthy control group $91.0 \mathrm{ng} / \mathrm{L}(52.4-119.7 \mathrm{ng} / \mathrm{L}), \mathrm{P}<0.01$.

Conclusions: For IPF with PD-L1 expression on alveolar macrophages, further studies are necessary to elucidate this phenomenon. Serum sPD-1/PD-L1 is easily detected in clinical practice and should be further evaluated as a potential prognostic or/and predictive biomarker in IPF.
\end{abstract}

Keywords: Idiopathic pulmonary fibrosis (IPF); membrane PD-L1 expression; soluble PD-L1

Submitted Jun 18, 2018. Accepted for publication Oct 18, 2018.

doi: $10.21037 /$ jtd.2018.11.16

View this article at: http://dx.doi.org/10.21037/jtd.2018.11.16

\section{Introduction}

Idiopathic pulmonary fibrosis (IPF) occurs in genetically susceptible individuals who are exposed to different environmental risks factors. It is associated with repetitive injury to the alveolar epithelium and subsequent activation of development pathways and aberrant immune and inflammatory signaling that results in the remodeling of the affected lungs. Patient with IPF are commonly older adults exhibiting heterogeneity in clinical course of the disease with a median survival of 2 to 3 years $(1,2)$.

IPF has common risk factors with cancer and significant 
similarities in the pathobiology process, both diseases having poor outcomes $(3,4)$.

Immune checkpoint PD-1 is one of the essential inhibitory molecules for controlling inflammatory response to injury in the normal lung tissues. Programmed death ligand-1/programmed cell death 1 (PD-L1/PD-1) axis represents a relevant negative feedback loop for maintaining immune homeostasis, but it is also of crucial importance for restricting tumour immunity $(5,6)$. Transforming growth factor- $\beta$ (TGF- $\beta$ ), a key growth factor in the pathogenesis of IPF, but also a significant pleiotropic cytokine for lung cancer, has the ability to modulate response to immune checkpoint inhibitors (5).

Tumors have the ability to escape from immunosurveillance and keep an immunosuppressive microenvironment which is stimulative for their unrestricted growth through multiple pathways. Among those is immunomodulation of T-cell response through downregulation of co-stimulatory molecules or enhancement of co-inhibitory molecules including immune checkpoints on $\mathrm{T}$ cells, other immune cells and tumor cells $(5,6)$. PD-L1 expressed on tumor and tumor-associated cells bounds to immune checkpoint PD-1 on cytotoxic T cells blocking their activation and proliferation.

Checkpoint inhibitor agents targeting PD-1/PD-L1 liberate antitumor $\mathrm{T}$ cells from the blockade enabling their further activation, proliferation and killing tumor cells. This cancer immunotherapy has revolutionized treatment and outcomes in several cancers including lung cancer.

Membrane-bound PD-1 and PD-L1 (mPD-1 and mPD-L1) also have soluble forms, the finding contributing to the complexity and multiplicity of the PD-1/PD-L1 (B7-H1/CD274) immunomodulation pathway (7-9).

Abundant mousse and human data released recently clearly point that checkpoint regulatory molecules are essential in some autoimmune diseases, not only in cancer $(10,11)$.

In a recently published study, RNA sequencing pointed to CD274 as significantly downregulated gene in human IPF lung tissue obtained by surgical biopsy (12).

We have undertaken this pilot study to evaluate which of the several cell types involved in IPF development and present in human IPF lung tissue exhibit immune checkpoint PD-L1 expression being positive for PD-L1 test, and to test if soluble forms of PD-L1, soluble PD-L1 (sPD-L1) can be detected in significant concentrations in blood of IPF patients which might be even considered potential diagnostic or prognostic biomarkers.

\section{Methods}

\section{PD-L1 expression in IPF tissue samples}

The same human IPF lung tissue samples (formalinfixed, paraffin-embedded, FFPE) of our 12 patients in which RNA sequencing pointed to CD274 as significantly downregulated gene, have been tested for PD-L1 expression. IPF was diagnosed in those patients by IPF Multidisciplinary Team, based on clinical-radiological criteria according to guidelines and histologic confirmation of UIP pattern. Lung tissue specimens were obtained by surgical biopsy, minor thoracotomy with biopsy, with the diameter of those biopsy samples ranging from 3 to $4.8 \mathrm{~cm}$.

PD-L 1 IHC $22 \mathrm{C} 3$ pharmDx, a qualitative immunohistochemical assay using monoclonal mouse antiPD-L1, Clone 22C3 has been used in the detection of PD-L1 protein in FFPE human IPF lung tissue samples. EnVision FLEX visualization system on Autostainer Link 48 was used.

PD-L1 protein expression was determined by using Tumor Proportion Score (TPS), which is the percentage of viable cells [in this study alveolar macrophages (AMs)] showing partial or complete membrane staining. The specimen was considered PD-L1 positive if TPS $\geq 50 \%$ of the viable AMs exhibited membrane staining at any intensity.

\section{Plasma samples preparation and storing}

Plasma samples for examination of sPD-L1 forms, PD-L1 (B7-H1/CD274) blood concentration determination, originated from our alive 23 patients with IPF who did not undergo surgical biopsy. IPF in those patients was made by IPF Multidisciplinary Team, based on clinical-radiological criteria according to guidelines. These plasma samples were separated by using BD Vacutainer lithium-heparin tubes (Becton Dickinson, Heidelberg, Germany) for blood collection and centrifuged for 15 minutes at $1,000 \times \mathrm{g}$, within 30 minutes of collection. Aliquoted plasma samples were stored at $-80{ }^{\circ} \mathrm{C}$ until analysis (maximally 6 months after the blood drawing).

\section{PD-L1 (B7-H1/CD274) blood concentration determination}

For the PD-L1 (B7-H1/CD274) determination in human plasma was used DuoSet ELISA system (R\&D systems Europe, Ltd. Abingdon, UK), as a sandwich enzyme-linked 
Table 1 Clinical characteristics of IPF patients undergone lung biopsy whose FFPE IPF lung tissue samples were tested for PD-L1 expression

\begin{tabular}{|c|c|c|c|c|c|c|c|c|c|}
\hline $\begin{array}{l}\text { Patients' } \\
\text { number }\end{array}$ & Gender & $\begin{array}{l}\text { Age at the time } \\
\text { of biopsy (years) }\end{array}$ & Diagnosis & \multicolumn{2}{|c|}{ Fibrosis stages } & $\begin{array}{l}\text { Macrophages } \\
\text { (number) }\end{array}$ & PD-L1+ [\%] & Finding & $\begin{array}{l}\text { Lymphocytes } \\
\text { PD-L1 expression }\end{array}$ \\
\hline 1 & $\mathrm{~F}$ & 65 & IPF & 3 & 3 & Large & $>50$ & + & PD-L1(-) \\
\hline 2 & $\mathrm{~F}$ & 49 & IPF & 3 & 3 & Moderate & $>1,<50[20]$ & + & PD-L1(-) \\
\hline 3 & $\mathrm{~F}$ & 38 & IPF & 2 & 2 & Small & $<1$ & - & PD-L1(-) \\
\hline 5 & $\mathrm{~F}$ & 39 & IPF & 3 & 3 & Large & $>50$ & + & PD-L1(+ up to $5 \%)$ \\
\hline 6 & M & 68 & IPF & 1 & 1 & Large & $>50$ & + & PD-L1(-) \\
\hline 7 & $M$ & 59 & IPF & 2 & 1 & Small & $<1$ & - & PD-L1(-) \\
\hline 8 & $\mathrm{~F}$ & 68 & IPF & 2 & 3 & Small & $<1$ & - & PD-L1(-) \\
\hline 11 & M & 37 & IPF & $3^{*}$ & 2 & Moderate & $>1[5]$ & + & PD-L1(-) \\
\hline 12 & M & 61 & IPF & $3^{*}$ & 3 & Moderate & $>1$ [10] & + & PD-L1(-) \\
\hline
\end{tabular}

${ }^{*}$, Honey comb lung. IPF, idiopathic pulmonary fibrosis.

immunosorbent assay (ELISA) specific for the human $\mathrm{B} 7-\mathrm{H} 1$. Results are reported in $\mathrm{ng} / \mathrm{L}$; in our hands the lower detection limit was $2.0 \mathrm{ng} / \mathrm{L}$, the upper limit of the test linearity was $1,250 \mathrm{ng} / \mathrm{L}$. According to manufacturer's data reference values for healthy people in heparin-plasma samples are 33-110 ng/L.

The study was approved by School of Medicine Belgrade University Ethics and Review Board of 06.06.2017 (No. 61206-2299/2-17).

\section{Results}

\section{PD-L1 expression in IPF tissue samples}

One of our goals was to explore mPD-L1 (clone 22C3) positivity in FFPE lung fibrosis tissue samples. Our study involved FFPE human lung tissue samples of twelve patients with IPF. Histological parameters included fibroblastic foci, as well as hypertrophic myofibroblast fibres, AMs or other present immune cells investigated for positivity to PD-L1 (Table 1).

We have found that PD-L1 expression on fibroblast and myofibroblast membrane was negative in all twelve cases of IPF. PD-L1 expression in IPF AMs was negative in three cases, but there was positivity to overexpression of PD-L1 in nine IPF cases. Only very few cells in the interstitium have shown discrete PD-L1 expression, but none of a membrane type (Table 1 and Figure 1).

\section{Soluble PD-L1 concentration in IPF patients and in bealthy subjects}

In order to compare lung disease biomarkers in IPF patients with healthy people, we have performed Kruskal-Wallis nonparametric ANOVA test followed by Mann-Whitney $\mathrm{U}$ test, as a post-hoc test, where appropriate. Twenty-three patients included had newly diagnosed IPF who did not undergo surgical lung biopsy (Table 2).

We have found elevated concentrations of sPD-L1 in the serum of IPF patients, significantly higher compared with healthy control group, $314.3 \mathrm{ng} / \mathrm{L}$ (117.7-483.1 ng/L) versus $91.0 \mathrm{ng} / \mathrm{L}(52.4-119.7 \mathrm{ng} / \mathrm{L})$, respectively $(\mathrm{P}<0.01)$. And not only that: $\mathrm{SPD}-\mathrm{L} 1$ levels in IPF patients were significantly higher compared with sPD-L1 levels in newly diagnosed non-small cell lung cancer (NSCLC) patients (study results in press). Results are presented in the Table 3. Moreover, our results showed that $\mathrm{SPD}-\mathrm{L} 1$ blood concentration is significantly higher in patients who had stage II IPF compared to IPF stage I group (estimated according to GAP score).

\section{Discussion}

IPF has common risk factors with cancer and significant similarities in the pathobiology process, regarding variety of genetic, epigenetic, cellular and molecular aspects and both 

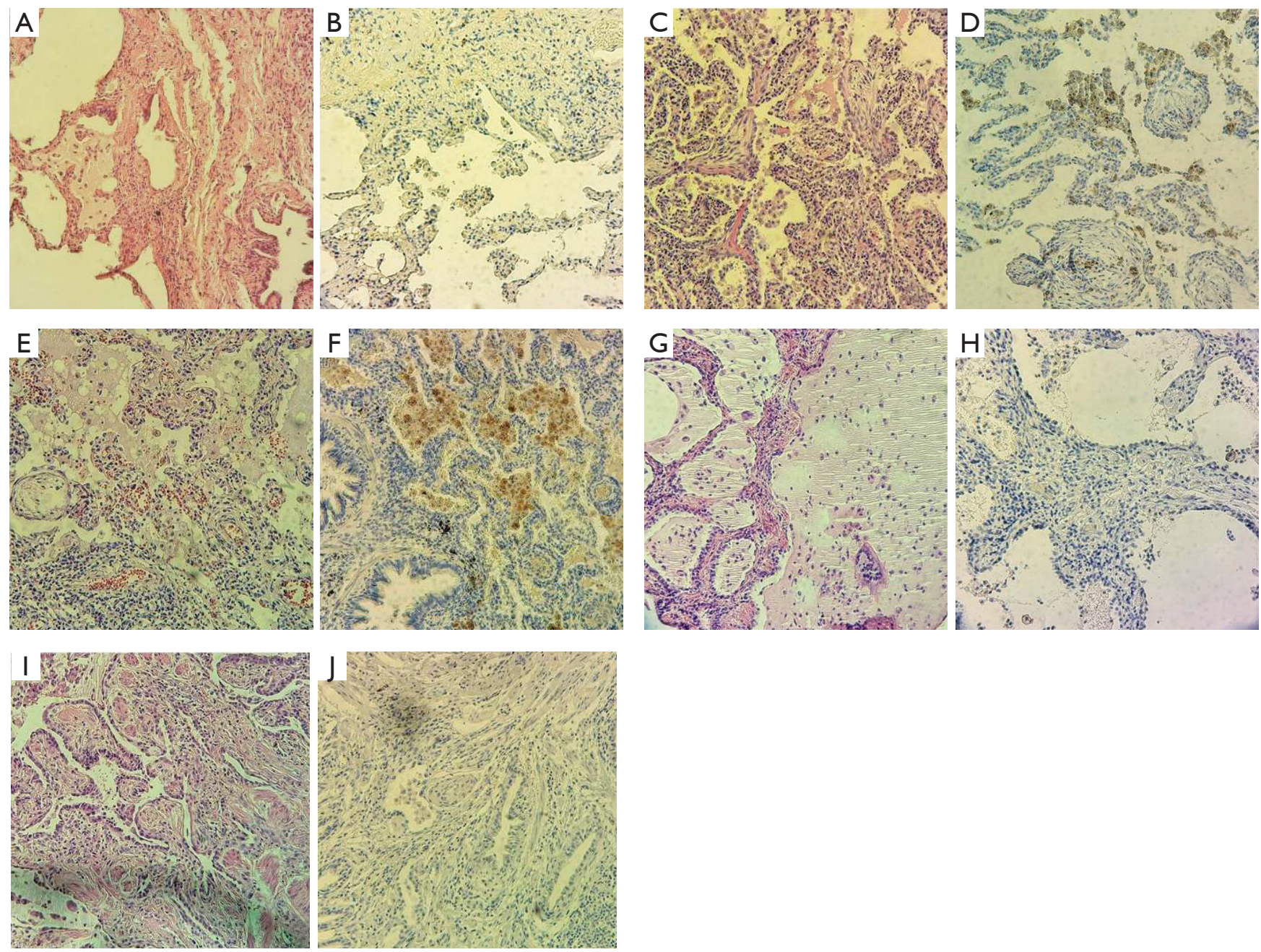

Figure 1 Programmed cell death-ligand-1 (PD-L1) expression in alveolar macrophages in different histological stages of usual interstitial pneumonia pattern (idiopathic pulmonary fibrosis). (A) Cystic remodeling of lung parenchyma ("honeycomb changes") (H\&E, ×20); (B) PD-L1 expression: >1\% positivity in alveolar macrophages; intensity of expression +1 ( $\times 20)$; (C) fibroblastic foci and moderate interstitial inflammation (H\&E, $\times 20)$; (D) PD-L1 expression: $>50 \%$ positivity in alveolar macrophages; intensity of expression +2 ( $\times 20)$; (E) fibroblastic foci and moderate interstitial inflammation (H\&E, $\times 20)$; (F) PD-L1 expression: $>50 \%$ positivity in alveolar macrophages; intensity of expression $+2(\times 20)$; (G) cystic remodeling of lung parenchyma $(\mathrm{H} \& \mathrm{E}, \times 20)$; (H) PD-L1 expression: >1\% positivity in alveolar macrophages; intensity of expression $+1(\times 20)$; (I) fibroblastic foci, smooth muscle proliferation and moderate interstitial inflammation $(\mathrm{H} \& \mathrm{E}, \times 20)$; (J) PD-L1 expression: $>50 \%$ positivity in alveolar macrophages; intensity of expression $+2(\times 20)$.

diseases have poor outcome $(3,4)$.

Immune checkpoint is one of the essential inhibitory molecules for controlling inflammatory response to injury in the normal lung tissues, and considered relevant in the pathogenesis of both IPF and lung cancer. Risk factors such as smoking and occupational and environmental exposures result in stimulus producing repetitive epithelial lung injury leading to abnormal wound healing predisposing to genetic mutations, and ultimately occurrence of IPF and lung cancer. Patients with IPF have an increased risk for lung cancer (4). On the other hand, pulmonary toxicity, fibrosing pneumonitis, as an immune-related adverse event of immunotherapy with anti-PD-1/PD-L1 agents is more common in patients with NSCLC than in patients with other tumor types, and clinical trials with anti-PD-1 and anti-PD-L1 agents excluded patients with underlying 
Table 2 Baseline characteristics and clinical course of 23 newly diagnosed IPF patients

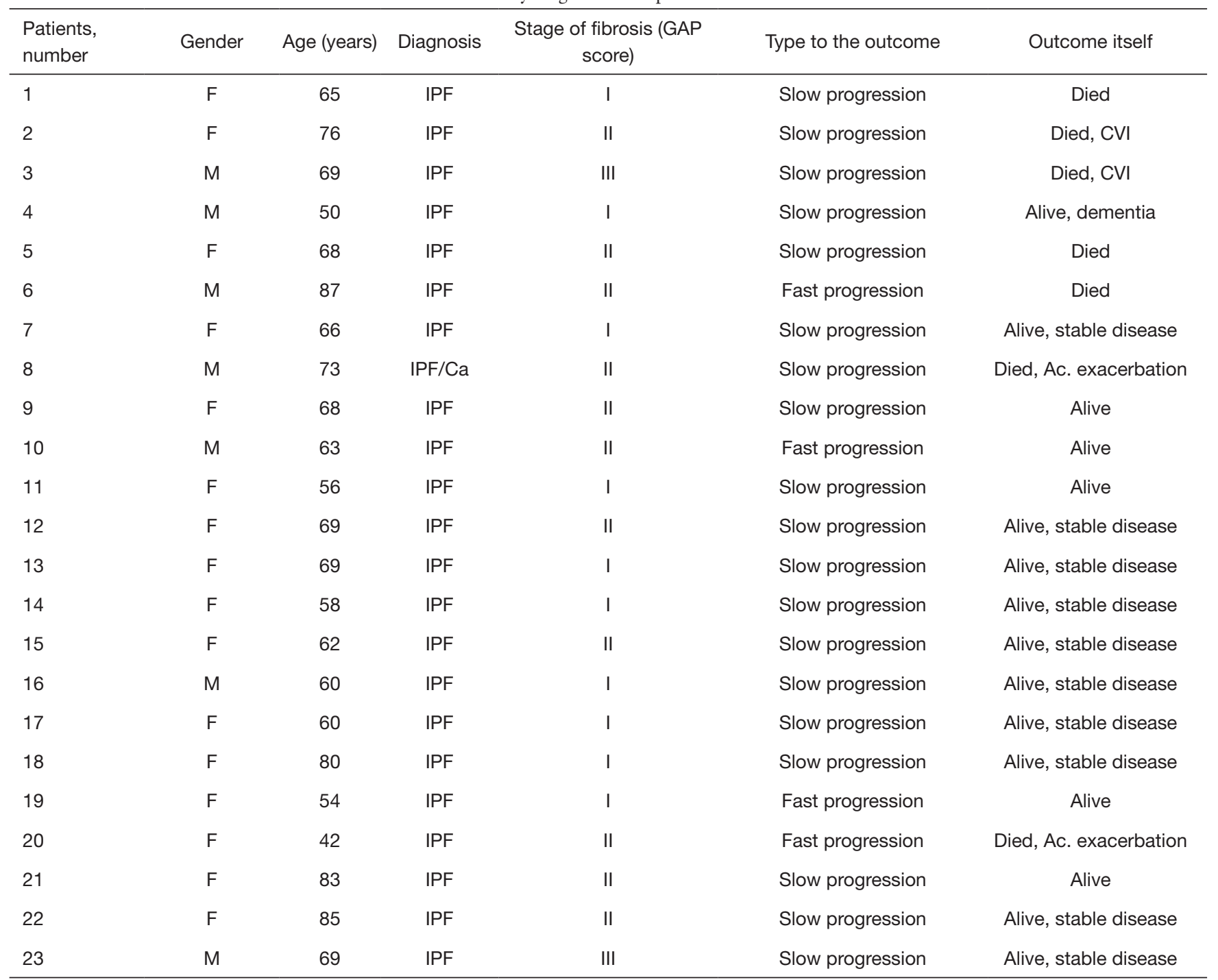

IPF, idiopathic pulmonary fibrosis; Ca, cancer; Ac., acute; CVI, cerebrovascular insult.

interstitial lung disease (ILD) (13).

Key finding in this pilot study is that mPD-L1 (over) expression was evidenced on AMs in 9 out of 12 IPF cases.

Under physiological conditions, AMs are critical for lung homeostasis and respiratory functions, not only surfactant clearance within the alveolar space (14), but also in directing pulmonary immune responses to pathogens and regulating tissue damage by suppressing immune responses (15). AMs colonize the lung within the first few days of life and have impressive self-renewal properties, the predominant mechanism for macrophage renewal being by expansion in situ $(16,17)$.

Although there are obvious differences among the namely M2 macrophages, regulatory macrophages, tumor associated macrophages (TAM) and myeloid derived suppressor cells (MDSC) subsets, they all exhibit immune suppressive activity $(14,18)$ and, consequently, when stimulated, macrophages adopt context-dependent phenotypes that either promote or inhibit host antimicrobial defense, anti-tumour immunity and inflammatory responses. There is evolving evidence that macrophages represent a spectrum of activated phenotypes rather than discrete stable subpopulations (18). Numerous studies have documented their plasticity, with macrophages switching from one functional phenotype to another in response to the variety of microenvironmental signals (18). 
Table 3 sPD-L1 concentration in sub-groups according to IPF stage

\begin{tabular}{ll}
\hline \\
Parameter
\end{tabular}

sPD-L1 (ng/L) 162.4 (85.4-263.7) 303.6 (249.2-572.5) 0.025

Data are presented as median values $\left(50^{\text {th }}\right.$ percentile) and $\left(25^{\text {th }}\right.$ $75^{\text {th }}$ percentile); $\mathrm{P}$ value from the Mann-Whitney $U$ test. IPF, idiopathic pulmonary fibrosis. SPD-L1, soluble programmed death ligand-1.

Since IPF is shown to be linked to the ageing process, AMs as long-lived cells have gained a lot of attention for potential profound implications for the clinical course and outcomes of IPF. The differentiation between recruited and resident macrophages seem to be important for the pathogenesis of fibrosis since the kind and function of produced cytokines can vary significantly between resident and recruited macrophages (16). AMs, in contrast to most other tissue macrophages, are shown to be dependent on transforming growth factor-b receptor (TGF- $\beta \mathrm{R}$ ) signaling. TGF- $\beta$ is crucial for the differentiation of fetal monocytes into preAMs during embryonic development, their maturation after birth, as well as for the homeostasis of adult AMs $(16,18)$. The source of TGF- $\beta$ is AMs themselves, indicative of an autocrine loop that promotes AM self-maintenance while TGF- $\beta$ are activated by AMs in an autocrine manner as well (15).

Macrophages are key regulators of fibrosis and they produce numerous pro-fibrotic soluble mediators, chemokines, and matrix metalloproteases. In the normal repair response to injury, macrophages acquire a phenotype which promotes fibroproliferation, so thus "pro-fibrotic" macrophages (also called M2) produce various mediators, pro-fibrotic cytokines and chemokines, including TGF- $\beta 1$, that directly activate fibroblasts and regulate the proliferation and survival of myofibroblasts, thus controlling extracellular matrix (ECM) deposition as well $(16,19)$. Pro-fibrotic macrophages also directly secrete matrix metalloproteinases (MMPs) and tissue inhibitors of metalloproteinases (TIMPs), regulators of inflammatory cell recruitment and ECM turnover (16). Although macrophages are necessary for the initiation and maintenance of fibrosis, some studies suggested that they were also required in the suppression, resolution and reversal of fibrosis and thus, with their ability to both initiate and inhibit fibrosis, macrophages are highly relevant for all stages of the fibrotic process. Based on numerous findings, macrophages seem to get distinct roles exhibiting a predominant phenotype dependent on the specific stage of fibrotic process $(16,19)$.

Our study results show that PD-L1 is overexpressed on AMs in IPF biopsy tissue samples, although not in the same extent in all of them. We have found that PD-L1 expression in IPF AMs was negative in three IPF cases, but there was positivity to overexpression of PD-L1 in nine IPF cases. Only very few cells in the interstitium have shown discrete PD-L1 expression, but not of a membrane type. PD-L1 expression on fibroblast and myofibroblast membrane was negative in all twelve cases of IPF.

It is well known that monocytes and macrophages function also as antigen-presenting cells sending costimulatory and coinhibitory signals to T cells (20), thus with ability to promote Th2 responses (21) which induce and activate TGF- $\beta 1$ in macrophages through an IL-13 - and MMP9-dependent mechanism (20). By expressing immuno-inhibitory ligands, such as PD-L1, macrophages can suppress $T$ cell immunity. Binding of PD-L1 to PD-1 expressed by T cells create an inhibitory signal that blocks the activity of $\mathrm{T}$ cells and this PD-L1/ PD-1 axis is an important negative feedback loop that ensures immune homeostasis, but also the way tumours effectively escape detection and suppress immune responses. PD-L1 high tumor-associated macrophages block the activity of tumor infiltrating cytotoxic T cells, antitumor $\mathrm{T}$ cells. Recognition of the role of PD-1/PD-L1 pathway in downregulating the activity of effector $\mathrm{T}$ cells within tumors and peripheral tissues has led to the development of cancer immunotherapy-checkpoint inhibitor therapy, that target PD-L1 expression on cancer and tumor-associated cells liberating antitumor $\mathrm{T}$ cells to proliferate and kill cancer cells (6).

Suppression of the cytotoxic $\mathrm{T}$ cell response may be directed by macrophages within the tumor microenvironment via expression of B7 family ligands (PD-L1, B7-H4), or indirect through release of IL-10 or recruitment of IL-10-expressing regulatory $\mathrm{T}$ cells $\left(\mathrm{T}_{\mathrm{Reg}}\right)$. In the latter, IL-10 suppresses the ability of dendritic cells to produce IL-12 and promote a Th1/cytotoxic T cell antitumor immune action (6). Response rates to treatment with checkpoint inhibitors in the clinical trials relate in part to PD-L1 expression in tumor stroma concordant with the functions of macrophages, and/or other stromal cells, in blocking anti-tumor $T$ cell responses $(6,22)$.

It should be noted that PD-1 is expressed not only in $\mathrm{T}$ cells, but also in TAMs, which become inactivated upon binding to PD-L1 expressed on tumor cells. TAMs with 
M2 phenotype (M2 TAMs) express PD-L1 as well as the ligand for the death receptor, FAS, that inactivates $\mathrm{T}$ cells and triggers their death. Treatment with the checkpoint inhibitor enables activation of cytotoxic $\mathrm{T}$ cells, but also increases phagocytic ability of TAMs against tumor cells. PD-1-positive TAMs show reduced phagocytic ability compared with PD-1 negative TAMs, further diminished if co-existent tumor cells express PD-L1 $(23,24)$.

The novel finding on M2 TAMs expressing PD-1 and PD-L1 and its implications (23), may contribute to the knowledge about the roles of the PD-1/PD-L1 pathway not only in the tumor but also in other immune microenvironment involving different immune cells. We could think of similar scenario with this novel finding in immuno-oncology translated from lung cancer to IPF, speculating how immune cells, especially monocytes and macrophages, foster fibrosis through molecular processes other than known as "proinflammatory" pathways.

Similar to TAMs, IPF macrophages may display two phenotypes, inflammatory M1 and a predominant alternatively activated M2 phenotype. This M2 phenotype exhibits marked expression of arginase, chitinase-like and resistin-like molecules $(24,25)$ and ability to promote secretion of fibroblast growth factors (including PDGF and FGF2), profibrotic cytokines including TGF- $\beta$ and interleukin (IL)-13 (26) and plasminogen activator inhibitor (PAI)-1 and MMPs (24). Moreover, recently shown in murine models of lung fibrosis, fibrocytes have no role in the collagen deposition (27) thus suggesting that macrophages (or their monocyte precursors) are of crucial importance in IPF (28).

Lung-resident macrophages may exhibit significant phenotypic plasticity that may be dependent on different stimuli or a microenvironment and thus M1/M2 phenotypes are considered more as a dynamic spectrum of activation potentially explaining some of the heterogeneity of IPF $(16,29,30)$.

When tissues are damaged, inflammatory mediators are released, macrophages often display an inflammatory M1-like phenotype, among other activities secrete MMPs such as MMP2 and MMP9 that help to degrade the ECM and enable the influx of inflammatory cells to the site of tissue injury $(16,28,29,31)$.

If the tissue-damaging factor persists, activated M1 cells can further exacerbate the inflammatory response by different mechanisms, leading to profound tissue damage. When the inflammatory stimulus is discontinued, M1 cell activation decreases, subsequent conversion of the immune response into a wound healing response occurs, with the accumulation of M2 macrophages that foster healing process and fibrosis through the secretion of MMPs (MMP12), TIMP1, growth factors and cytokines such as TGF- $\beta 1$. In the final phase, macrophages take on a regulatory/suppressive phenotype, which is featured by the expression of PD-L2 and IL-10 among others, which have all been shown to restore homeostasis while suppressing fibrotic process, partly by blocking $\mathrm{T}$ cell proliferation and collagen synthesis by activated myofibroblasts $(16,29)$.

Regarding our study findings that mPD-L 1 is overexpressed only in AMs in IPF biopsy tissue samples, it might be speculated that PD-L1 positive AMs have regulatory/suppressive phenotype with reduced degree of phagocytosis (as is the case with PD-L1-positive TAMs) and might reflect a specific IPF phenotype failing to limit the development of fibrosis and restore homeostasis.

Heterogeneity in the inciting stimuli, genetic predisposition and signaling mechanisms that promote profibrotic cell phenotypes may, like cancer, contribute to different clinical courses observed in patients with IPF that reflect different IPF Phenotypes. Thus, IPF with AMs PDL1 overexpression might be a specific IPF phenotype, or at least a particular IPF development phase. If so, then the approach to IPF, and fibrotic disease in general, may benefit from a similar precise medical approach.

Those novel finding on PD-1/PD-L1 axis and its implications (23), led to investigation of the potential roles of the PD-1/PD-L1 pathway both, in the tumor and other immune microenvironments. Co-inhibitory and costimulatory immune checkpoint receptors are necessary for full T-cell activation, and impaired tolerance causes the occurrence of majority of autoimmune diseases. There has already been evidence that PD-1 has a key "first-line" inhibitory impact on the majority of autoimmune diseases. However, genetic pattern influences the relative impact of their ligands B7.1, B7.2 and PD-L1, PD-L2, respectively. The influencing factors can also be the timing of ligand expression and interaction, as well as cell types expressing different one or other, and the specific cellular mechanisms underlying each disease $(10,11)$.

Emerging data suggest that immune checkpoint axes are dysregulated in chronic obstructive pulmonary disease (COPD) as well. Within diseased lungs, T-cells express the key receptor PD-1 and exhibit loss of their cytotoxic function, but there is also evidence of downregulation of the associated ligand PD-L1 on AMs. It is speculated that the resulting impact may be excessive T-cell inflammation due 
to acute infection, which may contribute to the pattern of COPD exacerbation and lung damage (32).

Pulmonary toxicity with anti-PD-1 agents is more common in patients with NSCLC than in patients with other tumor types, which is possibly due to impaired immune tolerance resulting from smoking-induced changes in the normal lungs. It is postulated that removal of checkpoint controls leads to excessive T-cell activation, tissue inflammation and damage, but all possibly important underlying factors are not still known (13). The reason lung cancer patients with underlying mild IPF or other fibrotic ILD were excluded from clinical trials with immunotherapy for lung cancer, checkpoint inhibitors, was the fear of the IPF/ILD deterioration, having in mind sometimes life-threatening pulmonary toxicity as an immune-related adverse event to immunotherapy with PD-1/PD-L1 checkpoint inhibitors. There are however increasing number of case reports on lung cancer patients with concomitant mild IPF or other fibrotic ILD who were treated with anti-PD-1/PD-L1 agents with good disease control and a no IPF/fibrotic ILD deterioration, so elucidation of some kind of individual background or susceptibility to immune-related toxicity pneumonitis is needed in the future (33).

Recent studies have discovered that $\mathrm{mPD}-1$ and mPD-L1 also have soluble forms, and there are findings pointing to the profound complexity of the PD-1/PD-L1 axis (7). The soluble forms of PD-1 and PD-L1 (sPD-1 and sPD-L1) evaluated in the blood of cancer patients may originate from proteolytic cleavage of the membrane-bound form, or by translation of alternative spliced mRNA. Both immune and tumor cells can be sources of sPD-L1, but as it is a characteristic of myeloidderived cells, it seems that there is another regulatory mechanism controlling the release of sPD-L1, different from that of mPD-L1. Since no correlation was noted between tumor PD-L1 expression and sPD-L1 levels, this implies that the tumor microenvironment, including nonmalignant cells, might also produce sPD-L1 $(7,26)$.

The precise roles of these molecules have not been elucidated yet, but a number of ongoing studies are trying to estimate their significance in human malignancies and evaluate their potential diagnostic, or prognostic and predictive biomarkers. Recent findings suggest that the blood levels of sPD-1/PD-L1 might predict treatment response and outcomes in cancer patients $(3,7,8)$.

The data show that sPD-L1 is normally detected in human serum with the levels increasing with age (26). It has been shown that the production of sPD-L1 can be suppressed by MMP inhibitors. These findings might be the explanation for the high serum concentrations of PD-L1 soluble forms in IPF patients $(11,26)$. We have found significantly higher concentrations of sPD-L1 in the serum of IPF patients compared with healthy control group, 314.3 versus $91.0 \mathrm{ng} / \mathrm{L}$ respectively $(\mathrm{P}<0.01)$. It has also been noticed that sPD-L1 levels in IPF patients were significantly higher compared with sPD-L1 levels in newly diagnosed NSCLC patients (reported study results in press). The question of precise functions of SPD-L1 in IPF, as well as in cancer and other autoimmune diseases, must be raised. The sPD-1/PD-L1 seem to be unrecognized factor that participate in immune response as well, but at present, whether sPD-L1 can bind to PD-1, similarly to mPD-L1, and act as an inhibitory signal is not clear. The findings of in vitro experiments where PD-1 or PD-L1 fusion proteins are added cause remarkably increased T-cell proliferation with expression of mPD-1 or mPD-L1. It implies that the abilities of mPD-1 and mPD-L1 are upregulated by their soluble forms. There are some controversial results regarding prognostic impact of high serum concentrations PD-L1 in different tumors $(7,9,34,35)$.

Our study findings of elevated concentrations of sPD-L1 in the serum of IPF patients, are significantly higher compared with healthy control group, and sPD-L1 levels in IPF patients are significantly higher compared with also significantly elevated sPD-L1 levels in newly diagnosed NSCLC patients (reported study results). Above mentioned results, require further investigations in a larger patients' population. Accumulated data at present indicate that sPD-1 and sPD-L1, which can be easily estimated in clinical practice, may have very important roles in immunopathogenesis and potential to reflect treatment responses, not only in tumors, but in IPF and other autoimmune disorders as well. It may be speculated that sPD-1/PD-L1 might be new biomarkers with significant impact on potential treatment strategies including immunotherapy in variety of diseases.

This is a pilot study that has several limitations. Perhaps, the most prominent is a small number of human IPF lung tissue samples that has been included in this research, thus not allowing reliable evaluation of this novel findings and clear conclusions. Larger number of IPF tissue samples is needed for the further research in order to elucidate the significance of $\mathrm{PD}-\mathrm{L} 1$ expression in AMs in a complex molecular pattern of IPF, and the impact of PD-L1, 
PD-L2/PD-1 axis in IPF. Another limitation is that we have not investigated bronchoalveolar lavage (BAL) for PD-L1 expression in AMs and other cells, which is going to be the next step. A small number of IPF patients were included in the study which has shown the significantly elevated concentrations of sPD-L1 in the serum of IPF patients. Thus, there is a need for more confirmatory studies, validation and new studies comparing this potential biomarker in different interstitial diseases in order to define its prognostic and/or predictive role.

\section{Conclusions}

IPF with AMs PD-L1 overexpression might be a specific IPF phenotype, or just an IPF development phase. Many questions raised from similarities in the pathobiology of IPF and lung cancer need answers. Based on these similarities and common expression of a number of growth factors, some lung cancer drugs have already been translated into treatment option for IPF (Nintedanib). This is a pilot study of membrane immune checkpoint PD-L1 expression in human IPF lung tissue samples and of soluble sPD-L1 plasma concentrations in IPF patients. For IPF with PD-L1 overexpression of AMs, further studies are necessary in order to elucidate this phenomenon and to potentially consider investigating some new treatment modalities or drug combinations as well. As for sPD-1 and sPD-L1 accumulated data at present indicate that they can be easily detected in clinical practice, and more importantly may have significant roles in immune responses and prediction not only in tumors, but in IPF and other autoimmune disorders as well. It may be speculated that SPD-1/PD-L1 should be evaluated as new prognostic or/and predictive biomarkers to aid potential treatment strategies including immunotherapy in the variety of diseases.

\section{Acknowledgements}

Funding: This work was supported by the Ministry of Education, Science and technological development of Serbia (contract No. 175046, 2011-2016).

\section{Footnote}

Conflicts of Interest: The authors have no conflicts of interest to declare.

Ethical Statement: The study was approved by the Ethics
Review Board of School of Medicine, University of Belgrade (06.06.2017) (No. 61206-2299/2-17).

\section{References}

1. Raghu G, Collard HR, Egan JJ, et al. An Official ATS/ ERS/JRS/ALAT Statement: Idiopathic Pulmonary Fibrosis: Evidence-based Guidelines for Diagnosis and Management. Am J Respir Crit Care Med 2011;183:788-824.

2. Daccord C, Maher TM. Recent advances in understanding idiopathic pulmonary fibrosis. F1000Res 2016;5. doi: 10.12688/f1000research.8209.1.

3. Vancheri C. Idiopathic pulmonary fibrosis and cancer: do they really look similar? BMC Medicine 2015;13:220.

4. Antoniou KM, Tomassetti S, Tsitoura E, et al. Idiopathic Pulmonary Fibrosis and Lung Cancer: A Clinical and Pathogenesis Update. Curr Opin Pulm Med 2015;21:626-33.

5. Santarpia M, González-Cao M, Viteri S, et al. Programmed cell death protein-1/programmed cell death ligand-1 pathway inhibition and predictive biomarkers: understanding transforming growth factor-beta role. Transl Lung Cancer Res 2015;4:728-42.

6. Chen DS, Mellman I. Elements of cancer immunity and the cancer-immune set point. Nature 2017;541:321-30.

7. Zhu X, Lang J. Soluble PD-1 and PD-L1: predictive and prognostic significance in cancer. Oncotarget 2017;8:97671-82.

8. Sorensen SF, Demuth C, Weber B, et al. Increase in soluble PD-1 is associated with prolonged survival in patients with advanced EGFR-mutated non-small cell lung cancer treated with erlotinib. Lung Cancer 2016;100:77-84.

9. Okuma Y, Hosomi Y, Nakahara Y, et al. High plasma levels of soluble programmed cell death ligand 1 are prognostic for reduced survival in advanced lung cancer. Lung Cancer 2017;104:1-6.

10. Zhang Q, Vignali DA. Co-stimulatory and Co-inhibitory Pathways in Autoimmunity. Immunity 2016;44:1034-51.

11. Dai S, Jia R, Zhang X, et al. The PD-1/PD-Ls pathway and autoimmune diseases. Cell Immunol 2014; 290:72-9.

12. Vukmirovic M, Herazo-Maya JD, Blackmon J, et al. Identification and validation of differentially expressed transcripts by RNA-sequencing of formalin-fixed, paraffin-embedded (FFPE) lung tissue from patients with Idiopathic Pulmonary Fibrosis. BMC Pulm Med 2017;17:15. 
13. Naidoo J, Wang X, Woo KM, et al. Pneumonitis in Patients Treated With Anti-Programmed Death-1/ Programmed Death Ligand 1 Therapy. J Clin Oncol 2017;35:709-17.

14. Hussell T, Bell TJ. Alveolar macrophages: plasticity in a tissue specific context. Nat Rev Immunol 2014;14;81-93.

15. Kopf M, Schneider C, Nobs SP. The development and function of lung-resident macrophages and dendritic cells. Nat Immunol 2015;16:36-44.

16. Byrne AJ, Maher TM, Lloyd CM. Pulmonary Macrophages: A New Therapeutic Pathway in Fibrosing Lung Disease? Trends Mol Med 2016;22:303-16.

17. Yu X, Buttgereit A, Lelios I, et al. The Cytokine TGF- 13 Promotes the Development and Homeostasis of Alveolar Macrophages. Immunity 2017;47:903-912.e4.

18. Stout RD, Jiang C, Matta B, et al. Macrophages sequentially change their functional phenotype in response to changes in microenvironmental influences. J Immunol 2005;175:342-9.

19. Broekelmann TJ, Limper AH, Colby TV, et al. Transforming growth factor beta 1 is present at sites of extracellular matrix gene expression in human pulmonary fibrosis. Proc Natl Acad Sci U S A 1991;88:6642-6.

20. Chen L, Flies DB. Molecular mechanisms of T cell co-stimulation and co-inhibition. Nat Rev Immunol 2013;13:227-42.

21. Martinez FO, Sica A, Mantovani A, et al. Macrophage activation and polarization. Front Biosci 2008;13:453-61.

22. Ruffell B, Coussens LM. Macrophages and therapeutic resistance in cancer. Cancer Cell 2015;27:462-72.

23. Gordon SR, Maute RL, Dulken BW, et al. PD-1 expression by tumour-associated macrophages inhibits phagocytosis and tumour immunity. Nature 2017;545:495-9.

24. Suda K. Tumor-associated macrophages_-additional effectors at anti-PD-1/PD-L1 therapy? J Thorac Dis 2017;9:4197-200

Cite this article as: Jovanovic D, Roksandic Milenkovic M, Kotur Stevuljevic J, Markovic J, Ceriman V, Kontic M, Skodric Trifunovic V. Membrane PD-L1 expression and soluble PDL1 plasma levels in idiopathic pulmonary fibrosis-a pilot study. J Thorac Dis 2018;10(12):6660-6669. doi: 10.21037/ jtd.2018.11.16
25. Mantovani A, Biswas SK, Galdiero MR, et al. Macrophage plasticity and polarization in tissue repair and remodelling. J Pathol 2013;229:176-85.

26. Chen Y, Wang Q, Shi B, et al. Development of a sandwich ELISA for evaluating soluble PD-L1 (CD274) in human sera of different ages as well as supernatants of PD-L1+ cell lines. Cytokine 2011;56:231-8.

27. Horowitz JC, Osterholzer JJ, Marazioti A, et al. "Scarcinoma": viewing the fibrotic lung mesenchymal cell in the context of cancer biology. Eur Respir J 2016;47:1842-54.

28. O'Dwyer DN, Ashley SL, Moore BB. Influences of innate immunity, autophagy, and fibroblast activation in the pathogenesis of lung fibrosis. Am J Physiol Lung Cell Mol Physiol 2016;311:L590-601.

29. Wynn TA, Vannella KM. Macrophages in tissue repair, regeneration, and fibrosis. Immunity 2016; 44:450-62.

30. Tan SYS, Krasnow MA. Developmental origin of lung macrophage diversity. Development 2016;143:1318-27.

31. Weidenbusch M, Anders H. Tissue Microenvironments Define and Get Reinforced by Macrophage Phenotypes in Homeostasis or during Inflammation, Repair and Fibrosis. J Innate Immun 2012;4:463-77.

32. Wilkinson TMA. Immune checkpoints in chronic obstructive pulmonary disease. Eur Respir Rev 2017;26. doi: 10.1183/16000617.0045-2017.

33. Khunger M, Velcheti V. A Case of a Patient with Idiopathic Pulmonary Fibrosis with Lung Squamous Cell Carcinoma Treated with Nivolumab. J Thorac Oncol 2017;12:e96-7.

34. Zhang J, Gao J, Li Y, et al. Circulating PD-L1 in NSCLC patients and the correlation between the level of PD-L1 expression and the clinical characteristics. Thorac Cancer 2015;6:534-8.

35. Ding Y, Sun C, Li J, et al. The Prognostic Significance of Soluble Programmed Death Ligand 1 Expression in Cancers: A Systematic Review and Meta-analysis. Scand J Immunol 2017;86:361-7. 